\title{
FOLGEN VON COVID 19 IN GESCHMACK UND GERUCH: EINE KURZE BIBLIOGRAPHISCHE ÜBERSICHT
}

\section{LITERATURISCHE REZENSION}

CHAVES, leda Bezerra ${ }^{1}$, FECURY, Amanda Alves², OLIVEIRA, Euzébio de ${ }^{3}$, DENDASCK, Carla Viana ${ }^{4}$, DIAS, Claudio Alberto Gellis de Mattos ${ }^{5}$

CHAVES, leda Bezerra. Et al. Folgen von COVID 19 in Geschmack und Geruch: eine kurze bibliographische Übersicht. Revista Científica Multidisciplinar Núcleo do Conhecimento. Jahr. 06, Hrsg. 11, Vol. 01, S. 150-166. November 2021. ISSN: 2448-0959, Zugangslink: https://www.nucleodoconhecimento.com.br/gesundheit/folgen-von-covid-19, $\quad$ DOI: 10.32749/nucleodoconhecimento.com.br/gesundheit/folgen-von-covid-19

\section{ZUSAMMENFASSUNG}

Viren sind ein Lebewesen, das nicht durch zelluläre Struktur gebildet wird. Viren können durch direkten Kontakt mit infizierten Personen oder indirekt von kontaminierten Oberflächen übertragen werden. COVID 19, definiert als eine neue Art von Coronaviren, hat die Weltgesundheitsorganisation (WHO) Anfang $2020 \mathrm{zu}$ einer Krise der globalen öffentlichen Gesundheit erklärt. Angesichts der hohen Übertragbarkeit dieses Virus war es notwendig, Maßnahmen zu ergreifen, um Übertragung und Infektion zu verhindern. Symptome, die durch COVID-19 verursacht

\footnotetext{
${ }^{1}$ Student der Integrierten Technischen Hochschule für Lebensmittel, Institut für grundlegende, technische und technologische Bildung von Amapá (IFAP).

${ }^{2}$ Biomedizin, PhD in Topischen Krankheiten, Professor und Forscher des Medical Course of Macapa Campus, Bundesuniversität of Amapa (UNIFAP).

${ }^{3}$ Biologe, PhD in Topical Diseases, Professor und Forscher des Physical Education Course, Bundesuniversität Para (UFPA).

${ }^{4}$ Theologe, PhD in Klinischer Psychoanalyse. Er arbeitet seit 15 Jahren mit Scientific Methodology (Research Method) in der wissenschaftlichen Produktionsberatung von Master- und Doktoranden. Spezialist für Marktforschung und Forschung mit Schwerpunkt Gesundheit. Doktorand in Kommunikation und Semiotik (PUC SP).

${ }^{5}$ Biologe, PhD in Theorie- und Verhaltensforschung, Professor und Forscher des Chemie-Studiengangs des Instituts für Grund-, Technik- und Technologiepädagogik (IFAP) und des Graduate Program in Professional and Technological Education (PROFEPT IFAP).
}

RC: 101287

Verfügbar in: https://www.nucleodoconhecimento.com.br/gesundheit/folgen-voncovid-19 
werden, sind in der Regel Fieber, trockener Husten, Kurzatmigkeit (Dyspnoe), Müdigkeit (Müdigkeit), Halsschmerzen, Kopfschmerzen und Durchfall. Andere Symptome sind in geringerem Maße Anosmie (Geruchsverlust), Hyposmie (verminderter Geruch) und Ageusie (Verlust des Geschmackssinns). Ziel war es, die letzten zwei Jahre auf die Folgen von COVID 19 in Geschmack und Geruch zu überprüfen. Eine kurze Literaturrecherche wurde in wissenschaftlichen Artikeln über die Folgen von COVID 19 in Geschmack und Geruch zwischen 2020 und 2021 auf Portugiesisch in der Google Scholar-Suchdatenbank durchgeführt. Frauen und Jugendliche sind am stärksten von den Folgen von Geschmack und Geruch betroffen, die durch COVID-19 verursacht werden. Es gibt keine spezifischen und wissenschaftlich nachgewiesenen Behandlungen für die verschiedenen Funktionsstörungen, aber die am meisten angezeigte ist die Praxis des olfaktorischen Trainings. Studien im Zusammenhang mit Funktionsstörungen sind begrenzt, vor allem in Brasilien und wenn sie als Folgeerscheinungen analysiert werden. Die Kontinuität der wissenschaftlichen Forschung ist unerlässlich, denn nur dieser Prozess wird in der Lage sein, die noch bestehenden Zweifel aufzuklären und geeignete Behandlungen für Menschen zu generieren, die an COVID-19 erkrankt sind.

Schlüsselwörter: Virus, COVID 19, Dysosmie, Dysgeusie.

\section{EINLEITUNG}

Viren sind ein Lebewesen, das im Gegensatz zu den anderen nicht von Zellen gebildet wird. Es hat normalerweise eine Nukleinsäure (die DNA oder RNA sein kann), die von einer Protein-"Kapsel" bedeckt ist. Einige sind immer noch von "Stücken" der Zellmembran bedeckt, die eine "Hülle" um sie herum bilden. Sie sind oft kleiner als eine Zelle und parasitieren sie, dringen in sie ein und nutzen ihre Strukturen, um mehr Viren zu produzieren (sie sind obligatorische intrazelluläre Parasiten) (Brandão, 2015; They, 2020). 
Viren können durch direkten Kontakt mit infizierten Personen oder indirekt von kontaminierten Oberflächen übertragen werden. Sie werden von einem Individuum zum anderen durch Wassertröpfchen in Form von Dampf übertragen, der in den Atem oder während des Husten- oder Niesprozesses freigesetzt wird. Kleinere Tröpfchen, die während dieser Prozesse zusammen mit Wasserdampf freigesetzt werden, werden in der Luft suspendiert und verbreiten das Virus über größere Flächen und länger. (Brasilien, 2021). In Kontakt mit einem dieser Übertragungsmittel dringen die Viren in die Schleimzellen der Atemwege und Digestórias ein (Stephens et al., 2009; Silva et al., 2020).

Zur Fortpflanzung binden die Viren an die Oberfläche der Zellmembran. Nach dieser Bindung kann es in die Zelle eindringen oder sein genetisches Material (DNA oder RNA) in sein Zytoplasma "injizieren". Sobald das genetische Material des Virus in den Zellen ist, wird es mehrmals kopiert, indem es die chemischen Reaktionen verwendet, die es machen kann. In ähnlicher Weise werden andere chemische Reaktionen der Zelle verwendet, um die "Abdeckungen" von Protein (Kapsiden) herzustellen. So werden neue Viren in einer Zelle montiert und, um auszugehen und in neue Zellen einzudringen, die Membran derjenigen brechen, die sie hergestellt haben (They, 2020; Silva et al., 2020a).

COVID 19, definiert als eine neue Art von Coronaviren, hat die Weltgesundheitsorganisation (WHO) Anfang 2020 zu einer Krise der globalen öffentlichen Gesundheit erklärt (Souza et al. 2020). Diese Viren mit einer hohen Übertragungsrate bestehen aus Hüllen von Lipiden und Proteinen (Membranstück einer Zelle) und Proteinkapsiden mit einem einzigen RNA-Band (Nukleinsäure) und mit äußerer Kronenform (Silva et al., 2020). Sie sind Teil der Familie der Viren, die Erkältungen zu schwereren Infektionen wie dem Middle East Respiratory Syndrome (MERS) und dem Severe Acute Respiratory Syndrome (SARS) erzeugen (Brasilien, $2021 ; 2021 a)$.

Angesichts der hohen Übertragbarkeit dieses Virus ist es notwendig, Maßnahmen zu ergreifen, um Übertragung und Infektion zu verhindern. Unter einigen dieser 
vorbeugenden Maßnahmen heben wir die Nicht-Dauerhaftigkeit an geschlossenen Orten und die Isolation infizierter Menschen hervor (Stephens et al., 2009).

Zu den Möglichkeiten, die als am effizientesten angesehen werden und eine Kontamination vermeiden (Prophylaxe), gehören: Händewaschen, soziale Distanzierung, Belüftung von Umgebungen und deren Sonneneinstrahlung, Reinigung von Gegenständen und Oberflächen, Verbot von Agglomerationen und die Verwendung von Masken (effiziente Blocker von verstreuten Atemtröpfchen, auch in reduzierten Entfernungen mit direktem oder indirektem Kontakt) (Garcia, 2020). Diese Kontrollmaßnahmen haben sich durch wissenschaftliche Analysen und Studien als wirksam erwiesen, um die Kontamination und Virusverbreitung zu reduzieren (Soares et al., 2021).

Symptome, die durch COVID-19 im Allgemeinen verursacht werden, sind Fieber, trockener Husten, Kurzatmigkeit (Dyspnoe), Müdigkeit (Müdigkeit), Halsschmerzen, Kopfschmerzen und Durchfall (Silva et al. 2020). Andere Symptome sind in geringerem Maße Anosmie (Geruchsverlust), Hyposmie (verminderter Geruch) und Ageusie (Verlust des Geschmackssinns) (Iser et al., 2020).

Folgeerkrankungen können definiert werden als eine Veränderung der Funktion von Zellen und/oder in der Form und Funktion von Organen, die einen dauerhaften Defekt verursachen oder nicht im Individuum (Filho, 2011; Vana und Schiozer, 2013).

$\mathrm{Zu}$ den verschiedenen Folgeerscheinungen, die bei Personen mit COVID19 gefunden wurden, gehören psychologische und biologische, wie Veränderungen der Atemwege, des Herz-Kreislauf-Systems, mögliche Haut- und neurologische Veränderungen (Depression und Angstzustände) (Estrela et al., 2021).

Eine Reihe von wissenschaftlichen Untersuchungen weist auf Langzeitschäden (nach 6 Wochen oder über 6 Wochen nach Beginn der Coronavirus-Symptome) im sensorischen System hin, wobei der Schwerpunkt auf olfaktorischen und gustatorischen Folgeerscheinungen liegt, z. B. Dysosmie (verzerrte Wahrnehmung von Gerüchen) und Dysgeusie (verzerrte Wahrnehmung des

RC: 101287

Verfügbar in: https://www.nucleodoconhecimento.com.br/gesundheit/folgen-voncovid-19 
Lebensmittelgeschmacks). Es kann auch Störungen des Glukosestoffwechsels (chemische Reaktionen), Hyperlipidämie (erhöhte Menge an Fett im Blut), Dysregulation des Fettstoffwechsels (chemische Reaktionen), posttraumatische Belastungsstörung (eine der Arten von Angststörungen) und betroffene Lungenkapazität, Schädigung der Leber (Leber) und des Nierensystems (Nieren) und möglicherweise im Fortpflanzungssystem (Sesapr, 2020; Grendene et al., 2021).

Geruchsstörungen haben eine quantitative Klassifizierung, bei der es Anosmie (keine Erkennung von Gerüchen), Hyposmie (Einschränkung bei der Erkennung von Gerüchen), Hyperosmie (Erhöhung der Fähigkeit, Gerüche zu erkennen) und Dysosmie (verzerrte Wahrnehmung von Gerüchen) gibt (Cardoso, 2018). Während quantitative Geschmacksstörungen als Hypogiesie (teilweise Geschmackserkennung), Hypergeusie (Erhöhung der geschmacklichen Wahrnehmungsfähigkeit), Ageusie (totaler Geschmacksverlust) und Dysgeusie (Geschmacksverzerrung) klassifiziert werden können (Barros et al., 2015; Gomes et al., 2020; Machado e Machado, 2020).

Die qualitative Unterklassifizierung von Dysosmie (olfaktorische Veränderung) ist vielfältig, unter den Beispielen findet man Parosmie (verzerrte und unangenehme Wahrnehmung von Gerüchen), Fantosmie (Geruchswahrnehmung in Abwesenheit von Geruchspartikeln) und Agnosmie (Unfähigkeit, verschiedene Gerüche zu klassifizieren) (Cardoso, 2018). Dysgeusie (geschmackliche Veränderung) hat eine qualitative Unterklassifikation, die auch diversifiziert ist, unter den Beispielen Parageusie (unzureichende Geschmackswahrnehmung aufgrund von Reizen) und Fantogeusie (unangenehme geschmackliche Wahrnehmung in Abwesenheit von Reizen) (Machado und Machado, 2020).

Trotz der Hinweise sind weitere Studien in Bezug auf die zahlreichen und vielfältigen Folgeerscheinungen durch Covid-19 erforderlich (Grendene et al. 2021), einschließlich betroffener Personen mit reduzierten Symptomen und asymptomatisch (Uzun, 2020). 


\section{ZIEL}

Machen Sie eine Literaturübersicht der letzten zwei Jahre über die Folgen von COVID 19 in Geschmack und Geruch.

\section{MEETHODE}

Eine kurze Literaturrecherche wurde in wissenschaftlichen Artikeln über die Folgen von COVID 19 in Geschmack und Geruch zwischen 2020 und 2021 auf Portugiesisch in der Google Scholar-Suchdatenbank durchgeführt. Es wurden die Schlüsselwörter (oder Deskriptoren) "Gustation und COVID19" und "Geruch und COVID 19" verwendet. Insgesamt wurden 1.777 Ergebnisse gefunden und 10 Artikel wurden verwendet, um diese kurze Überprüfung zu bilden. Dies ermöglichte es, die Überprüfung auf der Grundlage der aktuellsten akademischen Produktionen durchzuführen (Capes, 2012).

Artikel mit COVID-19-Inhalt und Folgeerscheinungen in Geschmack und Geruch wurden innerhalb der Frist der Überprüfung auf Portugiesisch verwendet. Artikel, die diesem Inhalt und/oder Zeitraum nicht entsprachen, wurden ausgeschlossen.

\section{ERGEBNISSE UND DISKUSSION}

Tabelle 01 zeigt die Anzahl der gefundenen Artikel und die Anzahl der Artikel, die innerhalb der methodischen Kriterien verwendet werden. 
Tabelle 1 - Anzahl der in der Datenbank gefundenen Artikel nach Deskriptoren und verwendeten Artikeln

\begin{tabular}{|c|c|c|c|c|}
\hline BASE DE DADOS & DESCRITOR & \begin{tabular}{|c|} 
NUMERO \\
ARTIGOS \\
ENCONTRADOS \\
\end{tabular} & $\begin{array}{c}\text { NUMERO } \\
\text { ARTIGOS } \\
\text { UTILIZADOS } \\
\end{array}$ & \begin{tabular}{|c|} 
AUTOR E ANO DOS \\
ARTIGOS \\
UTIIZADOS \\
\end{tabular} \\
\hline \multirow{10}{*}{ Google acadêmico } & \multirow{7}{*}{ Gustação e COVD19 } & \multirow{7}{*}{237} & \multirow{7}{*}{7} & PIMENTEL, 2020 \\
\hline & & & & $\begin{array}{l}\text { GRENDENE et al., } \\
2021\end{array}$ \\
\hline & & & & SANTOS et al., 2020 \\
\hline & & & & COSTA et al., 2020 \\
\hline & & & & SOUZA et al., 2021 \\
\hline & & & & $\begin{array}{c}\text { NOGUEIRA et al., } \\
2021\end{array}$ \\
\hline & & & & NUNES et al., 2020 \\
\hline & \multirow{3}{*}{ Olfato e COVID 19} & \multirow{3}{*}{1540} & \multirow{3}{*}{3} & KOSUGI et al., 2020 \\
\hline & & & & BRITO e SLVA, 2020 \\
\hline & & & & $\begin{array}{c}\text { LOPES e DE ABREU, } \\
2021\end{array}$ \\
\hline
\end{tabular}

RC: 101287

Verfügbar in: https://www.nucleodoconhecimento.com.br/gesundheit/folgen-voncovid-19 
Tabelle 02 - zeigt die Methoden, die in jedem für diese Überprüfung ausgewählten Artikel verwendet werden, die Ergebnisse und Schlussfolgerungen.

\begin{tabular}{|c|c|c|c|}
\hline $\begin{array}{c}\text { AUTOR E ANO } \\
\text { DOS ARTIGOS } \\
\text { UTILIZADOS }\end{array}$ & $\begin{array}{l}\text { TIPO DE } \\
\text { MÉTODO }\end{array}$ & RESULTADOS & CONCLUSÃO \\
\hline PIMENTEL, 2020 & $\begin{array}{l}\text { Pesquisa } \\
\text { qualitativa } \\
\text { descritiva, } \\
\text { revisão } \\
\text { bibliográfica da } \\
\text { literatura }\end{array}$ & \begin{tabular}{|} 
Estudos dassificaram as disfunções como: \\
anosmia/hiposmia ou ageusia e \\
hipogeusia/disgeusia. Pacientes tiveram os sintomas \\
disfuncionais tanto no olfato quanto na gustação. \\
Não são considerados danos permanentes.
\end{tabular} & $\begin{array}{l}\text { Identificação hiposmia, anosmia, fantosmia e } \\
\text { parosmia, com preval ência entre } 22,7 \% \text { a } 88,8 \% \text {, } \\
\text { associadas ou não hipogeu sia e ag eusia Futuros } \\
\text { estudos epi demi ológicos devem esclarecer como } \\
\text { esses sintomas são causados. }\end{array}$ \\
\hline $\begin{array}{l}\text { SANTOS et al } \\
2020\end{array}$ & $\begin{array}{c}\text { Revisão } \\
\text { Integrativa da } \\
\text { literatura }\end{array}$ & $\begin{array}{c}\text { Preval ência das disfunções sensoriais provocadas } \\
\text { pela COVD-19. Anosmia e disgeusia como } \\
\text { sintomas neurológicos mai s frequentes. Pacientes } \\
\text { que tiveram quadro leve a moderado de COVID-19. } \\
\text { Aumento da idade dos pacientes e quadros mais } \\
\text { severos da doença representam menos al terações. }\end{array}$ & $\begin{array}{l}\text { Sintese de informações de vários paises, } \\
\text { caracterizando as disfunções olfativas e gustativas } \\
\text { como sintomas iniciais. Repercu ssões após alta dos } \\
\text { pacientes. Necessários estudos sobre as } \\
\text { probl emáticas e investigações mais detalhadas. Os } \\
\text { distúrbios poderão ser estudados em um periodo de } \\
\text { tempo maior. }\end{array}$ \\
\hline $\begin{array}{l}\text { NOGUEIRA et al ., } \\
2021\end{array}$ & $\begin{array}{l}\text { Revisão } \\
\text { descritiva }\end{array}$ & $\begin{array}{c}\text { A infecção nos neurônios causa sintomas } \\
\text { neurológicos como anosmia. Não há explicação } \\
\text { exata acerca da ocorrência dos distúrbios olfatórios. } \\
\text { Danos no sistema nervoso central e epitélio nasal, } \\
\text { cau sam a di sosmia e a disgeusia. }\end{array}$ & $\begin{array}{l}\text { Os problemas olfativos e gustativo ocorrem } \\
\text { previamente em diferentes intensidades. Não há } \\
\text { evidências dientificas para o tratamento desses } \\
\text { distúrbios. É importante a realização de estudos } \\
\text { acerca do assunto a fim de que se melhore os } \\
\text { procedimentos no tratamento nos casos, } \\
\text { principalmente, nos casos com sequel as de longo } \\
\text { prazo. }\end{array}$ \\
\hline $\begin{array}{c}\text { COSTA et al., } \\
2020\end{array}$ & $\begin{array}{l}\text { Revisão } \\
\text { Sistemática }\end{array}$ & $\begin{array}{l}\text { Sintomas neurológicos que ocorrem no sistema } \\
\text { nervoso periférico causam as di sfunções olfativa e } \\
\text { gustativa. Pacientes com quadro leve a moderado } \\
\text { apresentaram hipogeusia e hiposmia, não há } \\
\text { registros do inicio, duração e resolução dos } \\
\text { sintomas. Os pacientes com perda de olfato tiveram } \\
\text { menor taxa de internação, compar ados com os } \\
\text { pacientes sem perda de olfato. }\end{array}$ & $\begin{array}{l}\text { Os distúrbios olfativos e/ou gustativos podem } \\
\text { ocorrer em intensidades variáveis, com inicio junto } \\
\text { com os sintomas prévios da infecção. Não há } \\
\text { evidências cientificas acerca do tratamento destes } \\
\text { distúrbios. }\end{array}$ \\
\hline
\end{tabular}

RC: 101287

Verfügbar in: https://www.nucleodoconhecimento.com.br/gesundheit/folgen-voncovid-19 


\begin{tabular}{|c|c|c|c|}
\hline $\begin{array}{c}\text { BRITO e SIVA, } \\
2020\end{array}$ & $\begin{array}{c}\text { Revisão da } \\
\text { literatura }\end{array}$ & $\begin{array}{l}\text { Associação de manifestações neurológicas e a } \\
\text { COVID-19. Entre elas os distúrbios do olfato e } \\
\text { paladar, como a hiposmia, a anosmia, a disgeusia, } \\
\text { hipogeusia e ageusia. Os mecanismos exatos da } \\
\text { neuroinvasão causada pela COVID-19 ainda são } \\
\text { desconhecidos. }\end{array}$ & $\begin{array}{l}\text { Ocorre uma inci dência maior de danos no si stema } \\
\text { nervoso em quadros agudos da virose. Entretanto a } \\
\text { classificação dos casos agudos e crônicos ainda hoje } \\
\text { não é consenso. São necessários mais estu dos sobre } \\
\text { as sequelas a longo prazo. }\end{array}$ \\
\hline $\begin{array}{c}\text { SOUZA et al., } \\
2021\end{array}$ & Relato de casos & $\begin{array}{c}\text { Ano smia nos pacientes é uma manifestação } \\
\text { sintomal ógica recorrente. Fundamental a descrição } \\
\text { de relatos de casos a fim de esclarecer as } \\
\text { ocorrências de sequel as gustativas olfativas com } \\
\text { objetivo de realializar o manejo da recuperação. }\end{array}$ & $\begin{array}{l}\text { As disfunções devem ser estudadas com maior rigor } \\
\text { e por maior periodo de tempo. A COVID-19 afeta } \\
\text { as células do epitélio olf ativo, possivelmente } \\
\text { alterando a neurotransmissão. }\end{array}$ \\
\hline $\begin{array}{l}\text { LOPES e DE } \\
\text { ABREU } 2021\end{array}$ & $\begin{array}{c}\text { Metodologia para } \\
\text { tratamento }\end{array}$ & $\begin{array}{c}\text { Possiveis danos neurológicos em nivel celular } \\
\text { decorrentes da COVID-19. Pacientes recuperados } \\
\text { ainda relatam alterações, que podem ser } \\
\text { irreversiveis, nos sentidos olf ativo e gustativo. }\end{array}$ & $\begin{array}{l}\text { As sequelas causadas pela doença podem ser de } \\
\text { cunho neurológicos, em nivel celular ou não. Isso } \\
\text { pode causar transtornos, sindromes e outras } \\
\text { variáveis futuramente. }\end{array}$ \\
\hline $\begin{array}{c}\text { NUNES et al., } \\
2020\end{array}$ & $\begin{array}{l}\text { Revisão } \\
\text { Integrativa }\end{array}$ & $\begin{array}{c}\text { Disfunções olfativas com preval ência entre } 30 \text { a } \\
88 \% \text { dos pacientes com COVD-19 englobados nos } \\
\text { diferentes estudos. Relados por pacientes com } \\
\text { quadros leves e moderados da doença. Os } \\
\text { transtornos como anosmia, hiposmiahipogeusia, e } \\
\text { disgeusia são sintomas frequentes nos pacientes } \\
\text { com COVID-19. }\end{array}$ & $\begin{array}{l}\text { O envolvimento do virus com o sistema nervoso é, } \\
\text { até então, inconclusivo. Entender os sintomas } \\
\text { neurológicos, principalmente em quadros mais } \\
\text { graves, auxilia nas intervenções e nos prognósticos } \\
\text { médicos. }\end{array}$ \\
\hline $\begin{array}{c}\text { GRENDENE et } \\
\text { al., } 2021\end{array}$ & $\begin{array}{c}\text { Revisão } \\
\text { bibliográfica }\end{array}$ & $\begin{array}{c}\text { Aponta que existem poucos estudos revisados sobre } \\
\text { a ocorrência ou prevalência das sequelas de longo } \\
\text { prazo da COVID-19. Há algumas evidências de que } \\
\text { disfunções como a disosmia e disgeusia são } \\
\text { sequel as de longo prazo associadas à COVID-19. A } \\
\text { mai oria não trabalha com periodos de } \\
\text { acompanhamento indi cativos de sequelas de longo } \\
\text { prazo. }\end{array}$ & $\begin{array}{l}\text { Não se sabe muito sobre estratégias para tratamento } \\
\text { para este virus. Pesquisas clíni cas estão sendo } \\
\text { realizadas para descobrir novos tratamentos. } \\
\text { Prevenção ainda é a melhor forma de lidar. }\end{array}$ \\
\hline $\begin{array}{c}\text { KOSUGI et al., } \\
2020\end{array}$ & $\begin{array}{l}\text { Quali quantitativa } \\
\text { por questionário }\end{array}$ & $\begin{array}{c}\text { Induidos } 253 \text { pacientes, de todas as regiões } \\
\text { brasileiras, uma maioria apresentou anosmia e } 13 \text {, } \\
2 \% \text { não tiveram recuperação da per da olfativa. } \\
\text { Alteração no paladar não está incluida na pesquisa. }\end{array}$ & $\begin{array}{l}\text { Anosmia apresenta menor taxa de recu peração e } \\
\text { duração mai or em pacientes por COVID-19 } \\
\text { positivos do que nos negativos. Hiposmia teve } \\
\text { maior taxa de recuperação do que a anosmia. }\end{array}$ \\
\hline
\end{tabular}

Durch Pimentel-Studien (2020), die auf 15 Publikationen basierten, die klinische Berichte in Europa Anfang 2020 beschrieben, wurden Fälle von Hyposmie, Anosmie, Fantosmie und Parosmie mit einer Prävalenz zwischen 22,7\% und 88,8\% identifiziert, die mit Hypogusie und Ageusie assoziiert oder nicht assoziiert sind.

Die Amplitude der sensorischen Symptomraten / Folgeerkrankungen ist auf den Unterschied zwischen den Studien sowie den Instrumenten, die von diesen verschiedenen wissenschaftlichen Forschungen verwendet werden, und sogar auf genetische Unterschiede in der Population zurückzuführen (Pimentel, 2020). Populationsunterschiede zeigen, dass die Häufigkeit des Auftretens von Geruchsgeschmacksanomalien beispielsweise in Populationen in Europa, Nordafrika, Südasien und Westasien höher ist als bei den niedrigsten Raten von

RC: 101287

Verfügbar in: https://www.nucleodoconhecimento.com.br/gesundheit/folgen-voncovid-19 
Patienten in Ostasien (Santos et al., 2020). Frauen und jüngere Menschen scheinen am stärksten von Olfaktor- und Geschmacksstörungen betroffen zu sein (Costa et al., 2020; Nascimento, 2020). Dieser Unterschied tritt wahrscheinlich auf, weil es einen Unterschied in der physiologischen Abwehrreaktion zwischen Altersgruppen und Gattungen der menschlichen Spezies gibt (Cardoso et al. 2020).

Die Studie von Santos et al. (2020) hebt hervor, dass olfaktorische und geschmackliche Dysfunktionen als erste Symptome auftreten und dass sie nach der Entlassung von Patienten größere Auswirkungen haben.

Die Krankenhausbehandlung von Patienten endet vor dem Auftreten von Folgeerkrankungen, wahrscheinlich weil es in Brasilien einen historischen Mangel an offenen Stellen und Gesundheitsfachkräften gibt. Die Zunahme des Krankenhausstroms von Patienten während der Pandemie scheint die Situation verschlimmert zu haben und eine spezifische und gründliche Nachsorge verhindert zu haben, da die Versorgung bereits prekär war (Santo et al. 2021).

Im integrativen Review von Nogueira et al. (2021) wurde festgestellt, dass olfaktorische und geschmackliche Probleme zuvor bei unterschiedlichen Intensitäten auftreten. Es gibt keine wissenschaftlichen Beweise für die Behandlung dieser Störungen.

Costa et al. (2020) identifizierten, dass olfaktorische und/oder geschmackliche Störungen bei unterschiedlichen Intensitäten auftreten können, wobei sie zusammen mit früheren Infektionssymptomen auftreten. Es gibt keine wissenschaftlichen Beweise für die Behandlung dieser Störungen.

Das menschliche Immunsystem variiert je nach einigen physischen und physiologischen Merkmalen (Cardoso et al., 2020). Die Intensität der Symptome kann aufgrund dieser Variationen auftreten. Bei Frauen beeinflussen die in Menstruationszyklen produzierten Hormone, die in der Antibabypille und bei der Behandlung von Hormonersatz (zur Bekämpfung von Wechseljahrsbeschwerden) 
vorhanden sind, das Abwehrsystem und unterscheiden es sich von dem von Männern (Rogero et al. 2021).

Das Alter ist auch ein Faktor für den Unterschied im Auftreten von Symptomen. Im Gegensatz zu Costa et al. (2020) und Nascimento (2020) scheint ein höheres Alter anfälliger für die durch das Virus verursachten Symptome zu sein. Ein älterer Darm hat typischerweise eine geringere Aufnahme von Nährstoffen und Abwehrzellenproduktion. Der Unterschied im Konzept zwischen den Autoren kann mit der spezifischen Art des Symptoms zusammenhängen (Rogero et al., 2021).

Stress beeinflusst auch die Immunabwehrfunktion. Das Nervensystem beeinflusst die Produktion und Freisetzung von Hormonen, die diese Funktion vermitteln können (Rogero et al., 2021). Jüngere Menschen können aufgrund ihrer kürzeren Erfahrung stärker von Stress betroffen sein.

Was die Behandlungen betrifft, die in der Wirkung gegen Olfaktor- und Geschmacksstörungen untersucht wurden, stellt die Literatur die Verwendung von Kortikosteroiden (entzündungshemmende Wirkung) des oralen und oralen, olfaktorischen Trainings, Vitamin A und Natriumcitrat vor. Es gibt andere therapeutische Alternativen, aber die oben vorgestellten sind förderlicher für die Wirksamkeit. Dennoch gibt es unzureichende oder ausreichende Evidenz über die Wirksamkeit dieser Behandlungen (Cardoso et al., 2020; Costa et al., 2020; Felipe et al., 2021; Neta et al., 2021).

Durch eine Literaturübersicht (Brito e Silva, 2020) glauben sie, dass es in akuten Fällen des Virus eine höhere Inzidenz von Schäden am Nervensystem gibt. Die Klassifizierung von akuten und chronischen Fällen ist jedoch noch kein Konsens.

Souza et al. (2021) kamen zu dem Schluss, dass Funktionsstörungen strenger und über einen längeren Zeitraum untersucht werden sollten. COVID-19 betrifft olfaktorische Epithelzellen und verändert möglicherweise die Neurotransmission. 
Die Studie von Lopes und De Abreu (2021) berichtet, dass die durch die Krankheit verursachten Folgen neurologischer Natur sein können, auf zellulärer Ebene oder nicht. Dies kann in Zukunft zu Störungen, Syndromen und anderen Variablen führen.

Olfaktorische und geschmackliche Funktionsstörungen treten im Allgemeinen in einer assoziierten Weise auf, da die olfaktorischen und gustatorischen Sinne (chemische Sinne, Wahrnehmen der Substanzen in der Nasen- und Mundhöhle) zusammenarbeiten und die Geschmacksempfindungen ausschließlich vom Geruch abhängen. Sensorische Empfindungen entstehen aufgrund der Interaktion von Molekülen mit den Geruchs- und Geschmacksrezeptoren. Geschmacksrezeptoren (gefunden in Regionen der Zunge, des Gaumens, des Rachens, der Epiglottis und der Speiseröhre) sind dafür verantwortlich, geschmackliche Informationen durch Nervenimpulse (Kommunikation zwischen Zellen, Neuronen) an das zentrale Nervensystem zu senden. Beim Geruch werden olfaktorische Informationen auch durch Neuronen, die durch elektrische Ströme (Synapsen) kommunizieren, an das zentrale Nervensystem übertragen (Neto et al., 2011; Oliveira, 2014).

Das COVID19-Virus verursacht Verletzungen von olfaktorischen Nervenzellen, da sich im Nasenepithel eine hohe Menge an Enzymen befindet, die die Diffusion und Replikation erleichtern. Als Reaktion auf eine Infektion "aktiviert" das Immunsystem das Abwehrsystem durch Cytosine (Proteine), die sogenannte zytopathische Wirkung, die den Neurotransmissionsprozess beeinträchtigen und einen Olfaktorverlust verursachen kann. Dies geschieht auch beim Geschmack, wo der Entzündungsprozess (zytopathische Wirkung) das Leben der Rezeptorzellen der Geschmacksknospen verringert (Cardoso et al. 2020; Felipe et al., 2021)

Wissenschaftliche Analysen zeigen die Wahrscheinlichkeit, dass das Virus das Nasenepithel erreicht, nicht direkt Neuronen. Die Zellen des Nasenepithels behalten das sogenannte lonengleichgewicht des Schleims, auf das Neuronen angewiesen sind, um die Synapsen bis zum zentralen Nervensystem zu bilden. Wenn dieses Gleichgewicht beeinträchtigt ist, funktioniert der Geruch nicht mehr so, wie er sollte, es kann zu Schäden an olfaktorischen Neuronen kommen. Dennoch gibt es keinen 
Beweis dafür, ob Anosmie direkt aus dem Virus resultiert oder ob es das Ergebnis einer immunologischen Reaktion ist (Nishioka, 2020).

Im gustatorischen Sinne sind gustatorische Rezeptorzellen nicht vom Virus betroffen, sondern die Stützzellen, was die Fälle von Geschmacksverlust erklären kann. Das olfaktorische Epithel hat die Fähigkeit, sich zu regenerieren, das heißt, Anosmie gilt als reversibel, jedoch können Patienten mit langsamer Genesung von dieser Dysfunktion eine andere Störung, Parosmie, aufweisen, bei der die Aromen (süß, bitter, salzig, sauer) wiederhergestellt werden, aber bestimmte Gerüche und Aromen unangenehm werden (Nishioka, 2020).

Nunes et al. (2020) fanden in ihrer Überprüfung heraus, dass die Beteiligung des Virus am Nervensystem bis dahin nicht schlüssig ist. Das Verständnis neurologischer Symptome, insbesondere bei schwereren Erkrankungen, hilft bei Interventionen und medizinischen Prognosen.

Die Beteiligung des Virus am Nervensystem scheint nicht schlüssig zu sein. Derzeit gibt es zwei Haupttheorien, die die neurologischen Auswirkungen von COVID-19 auf den Menschen erklären, die erste entspricht der Wirkung des Virus bei der Immundysregulation, wie der Überproduktion von Cytosinen, und verursacht somit neurologische Symptome. Zeigt die Möglichkeit an, dass sich das Virus durch Blut und Nervenbahnen zum Nervensystem ausbreitet. Die zweite legt nahe, dass eine Infektion im zentralen Nervensystem durch periphere Neuronen auftritt, die mit dem Virus infiziert sind (Felipe et al. 2021).

Brito e Silva (2020), Pimentel (2020), Santos et al. (2020) und Nogueira et al. (2021) sind sich über das wenige Wissen, das heute existiert, einig und schlagen die Kontinuität von Studien zu den Symptomen von COVID 19 vor. Dies ist wichtig, weil es durch wissenschaftliche Studien möglich sein wird, die Ursachen der Mechanismen zu definieren, die geruchsgefährdende Veränderungen verursachen und eine Grundlage für die Erforschung geeigneter Behandlungen für die Betroffenen 
bieten (Nishioka, 2020; Santos et al., 2020; Felipe et al., 2021; Lima et al., 2021; Oliveira et al., 2021).

In der Literaturübersicht von Grendene et al. (2021) deutet die Schlussfolgerung darauf hin, dass nicht viel über Strategien zur Behandlung dieses Virus bekannt ist. Aus diesem Grund wird klinische Forschung betrieben, um neue Behandlungen zu entdecken. Prävention ist auch heute noch der beste Weg, um mit Infektionen umzugehen.

Präventionsmaßnahmen gegen das Virus umfassen: Händehygiene oft, durch Waschen mit Wasser und Seife (Brechen der Lipidwand des Virus und tötet es) und / oder Verwendung von 70\% Ethylalkohol, Vermeiden der Hände mit Augen, Nase und Mund, Üben der Atemwegskennzeichnung (Pflege beim Husten oder Niesen), Tragen von Gesichtsmasken (physische Barriere gegen hängende Tröpfchen), Social Distancing von mindestens einem Meter, Desinfektion von Gegenständen und Oberflächen, symptomatische Isolation, Ästhetisierung in Innenräumen, Händeschütteln und Umarmungen vermeiden und sozialen Kontakt vermeiden. Alle sind nach wissenschaftlicher Grundlage indiziert (Stephens et al., 2009; Baptista und Fernandes, 2020; Garcia, 2020; Soares et al., 2021; Spdm, 2021)

In Bezug auf soziale Isolation weisen Studien auf ihre Wirksamkeit hin und helfen bei der Verringerung der Infektionsraten, sollte jedoch in Verbindung mit anderen Viruseindämmungsmeditationen durchgeführt werden (Who, 2021). Es gibt auch Beweise für die Belüftung geschlossener Stellen, wenn sie dem Sonnenlicht ausgesetzt sind und sie belüftet halten, da solche Umgebungen die Wasserdampftröpfchen von Husten, Niesen und dem Akt des Sprechens über Individuen enthalten (Opas, 2021). Die Verwendung von Masken ist wissenschaftlich belegt, es ist bekannt, dass diese PSA (persönliche Schutzausrüstung) eine physische Barriere, eine Wand, gegen das COVID-19-Virus ist (Salles et al., 2021). Darüber hinaus wird betont, dass Menschen auch nach der Impfung nicht aufhören sollten, die Präventionsmechanismen gegen das Virus zu verwenden / anzuwenden (Brasilien, 2021a). 
Kosugi et al. (2020) führten eine Online-Umfrage durch und kamen zu dem Schluss, dass Anosmie bei Covid-19-positiven Patienten eine niedrigere Genesungsrate und eine längere Dauer auf sich hat als bei negativen Patienten. Hyposmie hatte eine höhere Genesungsrate als Anosmie.

Bei Hyposmie, weil es sich um einen teilweisen Verlust handelt, ist es möglich, den Geruch zu "trainieren". Zu diesem Zweck werden Gerüche und ätherische Öle sechs Monate lang zweimal täglich verwendet. Bereits schwererer Verlust, Anosmie, wenn es keine sofortige Genesung gibt, ist die Behandlung medikamentös und verlängert (Pereira et al. 2020). Dies erklärt wahrscheinlich den Unterschied in den Wiederherstellungsraten beider Fortsetzungen.

\section{SCHLUSSFOLGERUNGEN}

Die Folgen von COVID-19 sind verschiedene Störungen, die die Sinnessinne (Geruch und Geschmack) in unterschiedlichem Maße beeinträchtigen und das Wohlbefinden und das tägliche Leben von Tausenden von Menschen beeinträchtigen.

Frauen und junge Menschen sind am stärksten von diesen Funktionsstörungen betroffen. Es ist nicht genau bekannt, wie das Virus neurologische Symptome bei Patienten verursacht und mit welchen Mitteln Funktionsstörungen auftreten. Es gibt auch keine spezifischen und wissenschaftlich nachgewiesenen Behandlungen für die verschiedenen Funktionsstörungen, aber die am meisten angezeigte ist die Praxis des olfaktorischen Trainings.

Studien im Zusammenhang mit Funktionsstörungen sind begrenzt, hauptsächlich in Brasilien und wenn sie als Folgeerscheinungen analysiert werden, dh nach einer bestimmten Zeit nach der Genesung von COVID-19-Patienten untersucht werden. Die Kontinuität der wissenschaftlichen Forschung ist unerlässlich, denn nur dieser Prozess wird in der Lage sein, die noch bestehenden Zweifel aufzuklären und geeignete Behandlungen für Menschen zu generieren, die COVID-19 entwickelt 
haben und mit einigen der verschiedenen Arten von Folgeerscheinungen, einschließlich sensorischer, zurückgelassen wurden.

\section{VERWEISE}

BAPTISTA, A. B.; FERNANDES, L. V. COVID-19, análise das estratégias de prevenção, cuidados e complicações sintomáticas. Revista Desafios, v. 7, p. 1-10, 2020.

BARROS, Ó. D. et al. Disgeusia: a propósito de um caso clínico. Revista Portuguesa De Medicina Geral E Familiar, v. 31, n. 4, p. 272-276, 2015.

BRANDÃO, R. E. L. Vírus e Retrovírus: Contributo para a Evolução das Espécies. 2015. 61p. (Mestrado). Faculdade de Ciências da Saúde, Universidade Fernando Pessoa, Porto PT.

BRASIL. Coronavírus - Como é transmitido? , Brasília DF, 2021. Disponível em: < https://www.gov.br/saude/pt-br/coronavirus/como-e-transmitido >. Acesso em: 06 set 2021.

- Doenças ocasionadas por vírus respiratórios emergentes, incluindo o COVID-19. Brasília DF, 2021a. Disponível em: < https://www.unasus.gov.br/cursos/curso/46164 >. Acesso em: 06 set 2021.

BRITO, W. G. F.; SILVA, J. P. D. O. Impactos neuropatológicos do COVID-19. Brazilian Journal of Health Review, v. 3, n. 3, p. 4227-4235, 2020.

CAPES. Treinamento no uso do Portal de Periódicos. Brasilia DF, 2012. Disponível em: < https://www.fca.unesp.br/Home/Biblioteca/portal-capes.pdf >. Acesso em: 06 set 2021.

CARDOSO, A. R. Olfato como Marcador Biológico. 2018. 21p. (Mestrado). Universidade de Lisboa, Lisboa PT. 
CARDOSO, M. D. C. et al. Anosmia e disgeusia no paciente com coronavírus: revisão narrativa. REAS/EJCH, v. 46, p. 1-8, 2020.

COSTA, K. V. T. D. et al. Desordens olfativas e gustativas na COVID-19: uma revisão sistemática. Braz J Otorhinolaryngol., v. 86, n. 6, p. 781-792, 2020.

ESTRELA, M. C. A. et al. Covid-19: sequelas fisiopatológicas e psicológicas nos pacientes e na equipe profissional multidisciplinar. Brazilian Journal of Development, v. 7, n. 6, p. 59138-59152, 2021.

FELIPE, L. P. et al. Compreensão das manifestações neurológicas induzidas por infecções pelo novo coronavírus: uma revisão integrativa. REAID, v. 95, n. 36, p. e21137, 2021.

FILHO, N. D. A. O que é saúde? Rio de Janeiro RJ: Editora Fiocruz, 2011. 160p.

GARCIA, L. P. Uso de máscara facial para limitar a transmissão da COVID-19. Epidemiol. Serv. Saude, v. 29, n. 2, p. 1-4, 2020.

GOMES, D. R. D. P. et al. Avaliação do paladar de idosos e sua relação com estado nutricional e hábitos alimentares. Pan American Journal of Aging Research, v. 8, n. 1, p. 1-8, 2020.

GRENDENE, C. S.; GULO, R. B.; BETIOL, R. S. M. Coronavírus (covid-19): história, conhecimento atual e sequelas de longo prazo. Revista Corpus Hippocraticum, v. 1, n. 1, p. 1-14, 2021.

ISER, B. P. M. et al. Definição de caso suspeito da COVID-19: uma revisão narrativa dos sinais e sintomas mais frequentes entre os casos confirmados. Epidemiol. Serv. Saude, Brasília, v. 29, n. 3, p. 1-11, 2020.

KOSUGI, E. M. et al. Recuperação incompleta e tardia da perda súbita do olfato na COVID-19. Braz. j. otorhinolaryngol., v. 86, n. 4, p. 490-496, 2020. 
LIMA, J. H. C. et al. Covid-19 e os danos ao aparelho olfatório causando anosmia. Revista Científica Multidisciplinar, v. 2, n. 8, p. e28665, 2021.

LOPES, D. O.; DE ABREU, F. Eletroterapia IVL no tratamento de Covid-19 e sequelas no sistema nervoso central. Brazilian Journal of Development, v. 7, n. 4, p. $42332-423402021$.

MACHADO, A. L. M.; MACHADO, M. C. M. Disgeusia: Revisão de Literatura. 2020. 56p. (Graduação). Universidade de Uberaba, Uberaba MG.

NASCIMENTO, M. A. Alteração das funções sensoriais de olfato e paladar e seus correlatos clínicos e funcionais em indivíduos com Covid-19. 2020. 71p. (Mestrado). Universidade Federal do Rio Grande do Norte, Santa Cruz.

NETA, F. I. et al. Pathophysiology and possible treatments for olfactory-gustatory disorders inpatients affected by COVID-19. Current Research in Pharmacology and Drug Discovery, v. 2, p. 1-11, 2021.

NETO, F. X. P. et al. Anormalidades sensoriais: Olfato e paladar. Arquivos Int. Otorrinolaringol., v. 15, n. 3, p. 350-358, 2011.

NISHIOKA, S. D. A. A perda de olfato na COVID-19 já tem explicação - pelos menos em parte. 2020. Disponível em: < https://www.unasus.gov.br/especial/covid19/markdown/335 >. Acesso em: 30 out 2021.

NOGUEIRA, J. F. et al. Distúrbios olfatórios decorrentes de infecção por SARSCoV-2: fisiopatologia, fatores de risco e possíveis intervenções. Research, Society and Development, v. 10, n. 11, p. 1-7, 2021.

NUNES, L. T. D. et al. Principais Manifestações Neurológicas decorrentes do COVID-19: uma revisão integrativa. Revista Saúde Coletiva, v. 10, n. 59, p. 42484254, 2020. 
OLIVEIRA, A. A. D. et al. Análise dos principais fatores de risco preexistentes em pacientes diagnosticados com a COVID-19 no Amapá, Amazônia, Brasil. Revista Científica Multidisciplinar Núcleo do Conhecimento, v. 17, p. 56-72, 2021. Disponível em: < https://www.nucleodoconhecimento.com.br/saude/riscopreexistentes $>$.

OLIVEIRA, F. V. Aromas: contextualizando o ensino de Química através do olfato e paladar. 2014. 137 p. (Mestrado). Universidade de Santa Maria, Santa Maria RS.

OMS. Série de documentos de informação sintéticos do escritório regional da oms para a áfrica sobre a COVID-19. Africa, p. 1-6, 2021. Disponível em: < https://apps.who.int/iris/bitstream/handle/10665/338819/WHO-AF-ARD-DAK-102021-por. pdf?sequence=1\&isAllowed=y >. Acesso em: 30 out 2021.

OPAS. Roteiro para melhorar e garantir a boa ventilação de ambientes fechados no contexto da doença causada pelo novo coronavirus, COVID-19. Washington D.C., 2021. Disponível em: < https://iris.paho.org/handle/10665.2/53938 >. Acesso em: 01 out 2021.

PEREIRA, A. C. C. M. et al. Revisão bibliográfica: Anosmia no COVID-19. Revista Cien, v. 15, n. 2, p. 96-, 2020.

PIMENTEL, B. N. As disfunções olfativas e gustativas como apresentação clínica da COVID-19. Research, Society and Development, v. 9, n. 8, p. e64985072, 2020.

ROGERO, M.; NATACCI, L.; AMANCIO, O. Nutrição \& Imunidade. São Paulo SP: SBAN, 2021. 27p.

SANTO, D. M. N. D. E. et al. Desafios do enfermeiro do Centro Cirúrgico frente à pandemia da COVID-19 e transição de uma sala cirúrgica em unidade de terapia semi-intensiva. REAS, v. 13, n. 6, p. 1-6, 2021. 
SANTOS, I. H. A. et al. Disfunções olfativas e gustativas na COVID-19. Research, Society and Development, v. 9, n. 12, p. e42591211363, 2020.

SESAPR. Reabilitação do paciente pós tratamento de infecção por SARS- CoV-2. Curitiba $\quad 2020 . \quad$ DR, Disponível em: < https://www.saude.pr.gov.br/sites/default/arquivos_restritos/files/documento/202012/Nota\%20orientativa\%20n\%C2\%BA\%2053\%20reabilita\%C3\%A7\%C3\%A3o.pdf >. Acesso em: 09 set 2021.

SILVA, A. W. C. et al. Caracterização clínica e epidemiologia de 1560 casos de COVID-19 em Macapá/AP, extremo norte do Brasil. Research, Society and Development, v. 9, n. 8, p. 1-21, 2020a. Disponível em: < https://rsdjournal.org/index.php/rsd/article/view/5499/4641 >.

SILVA, A. W. C. et al. Perfil epidemiológico e determinante social do COVID-19 em Macapá, Amapá, Amazônia, Brasil. Revista Científica Multidisciplinar Núcleo do Conhecimento, v. 4, n. 4, p. 05-27, 2020. Disponível em: < https://www.nucleodoconhecimento.com.br/saude/covid-19-em-macapa >.

SOARES, K. H. D. et al. Medidas de prevenção e controle da covid-19: revisão integrativa. Revista Eletrônica Acervo Saúde, v. 13, n. 3, p. 1-11, 2021. Disponível em: < https://doi.org/10.25248/reas.e6071.2021 >.

SOUZA, F. D. S. et al. "Anarquia do paladar" e anosmia pós-COVID-19: Seriam danos permanentes? Relato de casos. Revista de Ciências Biológicas e da Saúde, v. 4, n. 1, p. 1-6, 2021.

SOUZA, K. O. et al. Covid-19 e o cenário atual da Cidade de Castanhal PA, Brasil. Research, Society And Development, v. 9, n. 8, p. 1-17, 2020. Disponível em: < https://rsdjournal.org/index.php/rsd/article/view/5717/4998 >.

SPDM. HMB explica por que a higienização das mãos auxilia na prevenção da Covid-19. São Paulo SP, 2021. Disponível em: < 
https://www.spdm.org.br/saude/noticias/item/3390-hmb-explica-por-que-ahigienizacao-das-maos-auxilia-na-prevencao-da-covid-19 >. Acesso em: 04 out 2021.

STEPHENS, P. R. S. et al. Virologia. In: MOLINARO, E. M.;CAPUTO, L. F. G., et al (Ed.). Conceitos e métodos para a formação de profissionais em laboratórios de saúde. Rio de Janeiro RJ: EPSJV, v.4, 2009. p.125 - 220.

THEY, N. H. Você sabe o que é um vírus? Microbiologando Porto Alegre RS, 2020. Disponível em: < https://www.ufrgs.br/microbiologando/voce-sabe-o-que-eum-virus/ >. Acesso em: 06 set 2021.

UZUN, A. C. D. S. B. Sequelas da Covid-19 vão além da fadiga, ressalta especialista. Campo Grande MS, 2020. Disponível em: < http://www.ms.gov.br/sequelas-da-covid-19-vao-alem-da-fadiga-ressalta-especialista/ >. Acesso em: 06 set 2021.

VANA, L. P. M.; SCHIOZER, W. Sequelas de queimaduras: nova classificação. Rev Bras Queimaduras, v. 12, n. 3, p. 192, 2013.

Eingereicht: November 2021.

Genehmigt: November 2021. 